\title{
Public Health and COVID-19: From Response to Recovery
}

\author{
James J. James, MD, DrPH
}

\section{$\mathrm{T}$} his past week being Public Health Week, we need to be aware of three active or looming Public Health Disasters (PHDs). The first, we are all too aware of, is COVID-19. In the U.S., as of April $17^{\text {th }}$ we have almost 700,000 cases of COVID-19, some 34,000 related deaths, and 330 million Americans paralyzed with fear-Americans just emerging from a severe seasonal flu outbreak with an estimated 24,000 additional deaths. Because of significant differences in testing and reporting and multiple other factors such as population size and density it is difficult to predict the medical burden of COVID-19 in a given area. However, given unchecked community transmission in a highly populated urban area one can predict a large number of hospitalized serious and critical cases that may well exceed the capacity of medical systems already stretched thin with the provision of necessary services to the populations they serve. This formula cannot help but produce excess mortality rates and such results have virtually crippled the healthcare systems of some of our largest metropolitan areas and have justifiably resulted in all out efforts to "flatten the curve" through the imposition of state-wide lockdowns as well as a number of socialdistancing restrictions of various degrees on public freedoms across the country. Given our understanding of COVID-19 and the rapidly increasing epidemic curves when these measures were instituted, the more drastic steps were the only interventions available to our government leaders charged with our health and protection.

Unfortunately, these interventions each come with a cost, and in the overall national interest we need to optimize our strategies to fully support our front-line medical systems while mitigating socio-economic damages. To better do this we need to assess and study, as best we can, the effectiveness of the interventions employed and guard against drawing false conclusions that are more representative of association, not causality. This is especially important at this time as we see some tapering off in new cases and new deaths for two reasons: 1) the conclusions we draw in the aftermath of COVID-19 will surely inform our response to the next pandemic event, and 2) the U.S. is almost universally imposing harsh social distancing interventions whereas in many areas of the country less drastic measures might well achieve the same objective with fewer negative socio-economic impacts. For example, a review of the global COVID-19 data by country presented at Worldometer (https://www. worldometers.info/coronavirus/) clearly demonstrates that in Korea, Japan, and many other countries where partial, risk targeted interventions were employed (see https://www.businessinsider.com/countries-on-lockdowncoronavirus-italy-2020-3 for a continuously updated list of worldwide restrictions), population medical outcomes are better than, or equal to those of nations that have imposed more extreme lockdowns. Furthermore, we have to realize that the complete lockdown of a city is not possible as necessary services, such as medical care, public safety, food distribution and security must be continued and will be ongoing potential sources of transmission to co-workers and family members. Add to this those involved in sustaining services at the national level, such as the military and the essential industrial base, and you have a porous lockdown at best.

The second PHD is the one we are self-inducing through shutting down our economy and shuttering our educational institutions. We are already seeing increased alcohol consumption and domestic abuse cases across the nation, most prominently among the newly unemployed, with our more socially and economically disadvantaged predictably impacted disproportionately. And as any student of history or world affairs can attest, continued socio-economic distress will produce predictable waves of mental illness, crime, suicide and a host of chronic diseases, and at the global level, can threaten political stability and promote conflict. Further inhibiting our ability to make informed decisions is the persistent lack of the necessary data to guide decision makers as they struggle with these competing priorities. We still cannot fully characterize the epidemiology of COVID-19 and there is a pressing need to measure the community prevalence of the infection, the ratio of susceptibles to non-susceptibles, and to better understand the mechanisms of transmissions. The tools to accomplish this are now becoming available and the sooner the scientific community can provide the necessary data, the sooner decision makers can tailor policies to them. This may prove to be even more important over the next several years as, given 
the level of penetration around the world, COVID-19 may well join other infectious diseases as being permanently imbedded in our eco-system.

The third PHD is more a fear than a reality but the deeper the socio-economic wound, the more probable the reality becomes. There is always another PHD on the horizon, and it remains in our interest to be prepared for it. When will the next major earthquake occur? What will the imminent hurricane season bring? Is there another novel infectious agent ready to emerge? What new conflicts will arise secondary to the effects of a world-wide recession, let alone a depression? When one or more of these events occur, where will the reserve capacity be to respond? There will be surges, yes, but that will be in global morbidity and mortality, not in relief, as our response to the current pandemic may leave us little reserve to respond effectively. It behooves us all to both mediate the medical impact of COVID-19 and simultaneously mitigate the associated socio-economic damages. In doing so, we will be better prepared for what is to come. The prescription for what I feel is a reasonable path forward requires a shift in perspective. While the extent to which social media have driven personal thinking about COVID-19, up to now the print and broadcast media have largely controlled the public narrative. They have, unfortunately, focused primarily on the overall numbers of COVID-19 cases and deaths without qualification, presented hyperbolic models of potential disease spread and impact, and have focused on emotionally charged individual cases as representative examples of the disease. This has fueled public fear and engendered political action and policies that, to date, have rightly addressed the medical response to the virus, but have done little to address the even more ominous socio-economic costs of a prolonged economic shutdown.

From a public health perspective we are faced with seemingly competing priorities; minimizing the current loss of life through public health interventions versus minimizing future lives lost by limiting those interventions. Compounding this conundrum, we cannot accurately quantify the lives lost under either construct. Therefore resolving the dilemma will involve achieving consensus from subject matter experts to define an optimization model that permits addressing both priorities simultaneously. This for me is a classic public health problem that is well suited to a risk management approach and as a nominal example I would offer the following.

A Rx for moving forward:

1) Turn down the noise level. We need to help people understand that a COVID-19 diagnosis is not a death sentence. Although it can be serious in younger age groups, for the great majority of healthy individuals under 65 , it is a relatively benign disease, while even in older age groups, the majority of those who contract the disease will survive. Linked closely to this is the need to better differentiate our case definition and reporting. The number of serious cases should be clearly distinguished from test positives in order to lessen the psychological impact of the constant headlining of big numbers.

2) Identify the individual and community risk factors as we do for all diseases. With COVID-19 the documented and consistent individual risk factors are age over 65 and preexisting medical conditions. Community risk factors are clearly population size and density, prevalence of test positives, mass gatherings, health care facilities and senior concentrations.

3) Define relative risk levels (such as low, moderate and high) based on the individual and community risk factors and tailor targeted interventions against those levels - classic risk assessment and risk management. Identify the appropriate authority level to implement the interventions. Given the size and complex make-up of the U.S., a one size fits all national standard is difficult to defend scientifically or logically. Even within our states, many of which could be standalone countries, the appropriate authority level responsible for risk assessment and response for any given community needs to be identified. State level health departments working in concert with city and county health counterparts could craft the needed risk-adjusted interventions for communities which, in turn, could focus on controlling their individual risk profiles. One of the few truisms in disaster medicine is that "all disasters are local;" it only follows that solutions must also be locally defined.

4) Use the above mentioned risk stratification methods and defined leadership structure to: a) insure the availability and appropriate distribution of supplies and equipment needed by the medical system to accomplish their patient care mission under what has become war-time conditions in too many of our hospitals; b) insure the funding and facilitation of the development, approval and deployment of pharmaceuticals, vaccines, and needed diagnostic and serological testing modalities for COVID-19 and future biologic threats; and c) regulate national and international commerce and travel in support of pandemic containment and mitigation strategies.

5) Most important, begin the recovery process. Adjust riskbased interventions to allow for the reopening of businesses and institutions in communities across the country once safe and feasible. This can begin the process of restoring jobs and healing the economy, thereby lessening the poor health outcomes associated with socio-economic hardships while optimizing our medical response to COVID-19. An added benefit would be diluting some of the rampant fear with a dose of hope.

This risk management approach to dealing with the current and impending disasters facing our nation will give Public Health the responsibility and authority to use the tools already at their disposal to truly protect the entirety of our population for this and succeeding generations of Americans. 